04

\title{
Изменение направления электрического ветра при наклоне игольчатого электрода относительно заземленной плоскости
}

\author{
() И.А. Елагин, Д.И. Бегаль, И.А. Ашихмин, Ю.К. Стишков
}

Санкт-Петербургский государственный университет

E-mail: i.elagin@spbu.ru

Поступило в Редакцию 14 июля 2016 г.

Проведено экспериментальное исследование структуры электрического ветра в системе электродов игла-плоскость. При фиксированном межэлектродном расстоянии варьировался угол наклона иглы. С помощью установки лазерной анемометрии получены характерные распределения скоростей. Показано, что узкая центральная струя электрического ветра существенно изменяет свое направление соответственно углу наклона коронирующего электрода.

DOI: 10.21883/PJTF.2017.02.44183.16410

Электрический (или ионный) ветер возникает во внешней зоне коронного разряда при подаче высокого напряжения на электрод с малым радиусом кривизны. Он обусловлен потоком ионов, вылетающих из чехла короны [1], размер которого невелик и обычно не превышает $100 \mu \mathrm{m}$. Вылетая из чехла, ионы обычно следуют по силовым линиям электрического поля, передавая свой импульс нейтральным молекулам воздуха. Скорость электрического ветра при этом значительно ниже скорости дрейфа ионов, поэтому конвективная составляющая ионного тока в воздухе мала.

В данной работе рассматривается одиночный игольчатый электрод, расположенный над заземленной пластиной. Проведено исследование положительной короны, чехол которой имеет облегающую форму и при высоких напряжениях охватывает не только головку иглы, но и область заострения. Плотность объемного заряда быстро спадает в направлении от иглы к плоскости [2], поэтому зона эффективного действия кулоновских сил обычно имеет размеры порядка $1 \mathrm{~mm}$. В этой области направление кулоновских сил определяется в основном геометрией коронирующего электрода и его расположением в пространстве. 
Поэтому, изменяя положение иглы относительно плоскости, можно управлять струей электрического ветра. Однако это утверждение требует экспериментальной проверки.

Необходимо отметить, что исследования, связанные с экспериментальным изучением характеристик электрического ветра в различных системах электродов, в том числе игла-плоскость, неоднократно проводились ранее другими авторами [1,3-7]. При этом во многих случаях варьировались такие параметры, как межэлектродное расстояние, напряжение, полярность и т.п. Однако во всех работах, в том числе для игольчатых и других электродов, имеющих определенное направление заострения, рассматривалось некоторое фиксированное взаимное расположение электродов (для системы электродов игла-плоскость по нормали). Подробная визуализация течений и пространственное распределение скорости электрического ветра приводятся достаточно редко (например, [5,6]) и используются обычно для сложных систем электродов, например при исследовании электрофильтров [7]. В работах $[8,9]$ было продемонстрировано влияние коронного разряда в системе электродов провод-плоскость на внешний поток, обтекающий пластину, и изучена возможность его регулирования.

В данной работе при постоянных напряжении, полярности и расстоянии между кончиком иглы и пластиной было проведено варьирование угла наклона иглы относительно нормали к плоскому электроду. Для визуализации течений и получения распределений скорости в области интереса с высоким пространственным разрешением использовалась современная установка лазерной анемометрии. Локальное изменение направления электрического поля в области, близкой к чехлу коронного разряда, которая вносит основной вклад в ускорение воздушных течений за счет действия кулоновской силы, привело к тому, что струя электрического ветра также изменяла направление соответственно наклону иглы.

Для проведения эксперимента была использована игла толщиной $1.2 \mathrm{~mm}$ с радиусом закругления кончика $0.3 \mathrm{~mm}$, расположенная на расстоянии $20 \mathrm{~mm}$ от алюминиевой пластины размерами $19 \times 10 \mathrm{~cm}$. На рис. 1 схематично показано, каким образом проводилось варьирование наклона иглы на угол $\alpha$ относительно нормального расположения. На коронирующий электрод подавалось фиксированное постоянное напряжение $10.4 \mathrm{kV}$ положительной полярности.

В области интереса с помощью PIV-установки (FlowMaster от компании LaVision) снимались картины распределения скоростей. Для

Письма в ЖТФ, 2017, том 43, вып. 2 


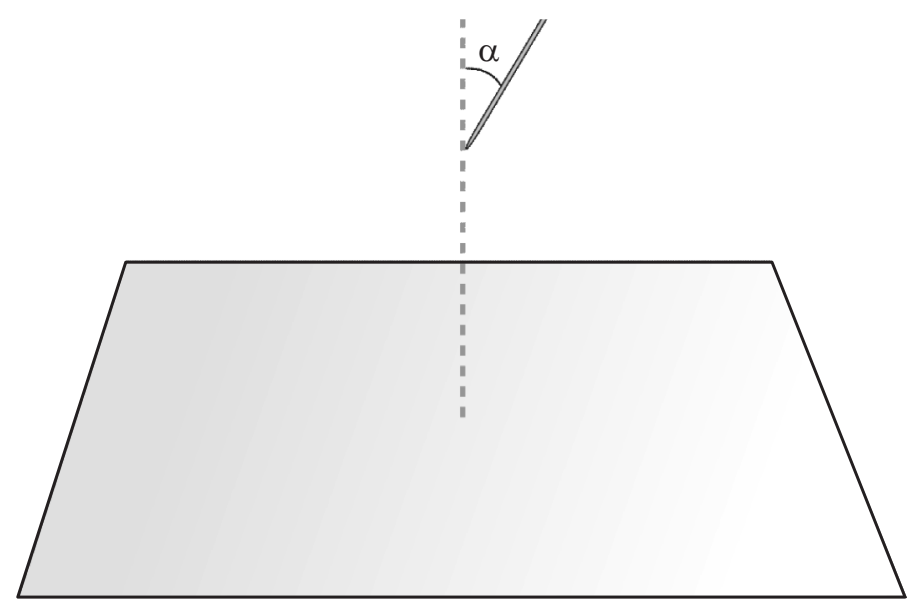

Рис. 1. Схема экспериментального макета.

визуализации течений использовался аэрозоль $\mathrm{C}_{26} \mathrm{H}_{50} \mathrm{O}_{4}$, частички которого имеют малые размеры и практически не влияют на основные физические характеристики рассматриваемого явления [4]. Специальная обработка последовательности кадров позволяет получить распределение скорости в плоскости съемки. На рис. 2 представлены распределения скорости течений в плоскости варьирования угла $\alpha$, проходящей сквозь иглу. Пунктиром обозначены отрезки, вдоль которых на рис. 3 будут приводиться соответствующие поперечные профили скорости. При стандартном расположении иглы по нормали к плоскому электроду (рис. 2,a) наблюдается характерная структура струйного течения с областями ускорения у острия иглы, равномерного течения в основной части межэлектродного промежутка и торможения у пластины, после соударения с которой струя расходится в стороны. Необходимо отметить высокие скорости получаемых течений (порядка $7 \mathrm{~m} / \mathrm{s}$ ), а также малую ширину струи (порядка $2 \mathrm{~mm}$, что более подробно видно на линейном графике профилей скорости на рис. 3). Размер зоны ускорения мал и составляет примерно $1-2 \mathrm{~mm}$.

При наклоне иглы относительно нормального расположения наблюдается изменение направления струи электрического ветра. В достаточно большом диапазоне угол наклона струи очень близко соответствует

Письма в ЖТФ, 2017, том 43, вып. 2 

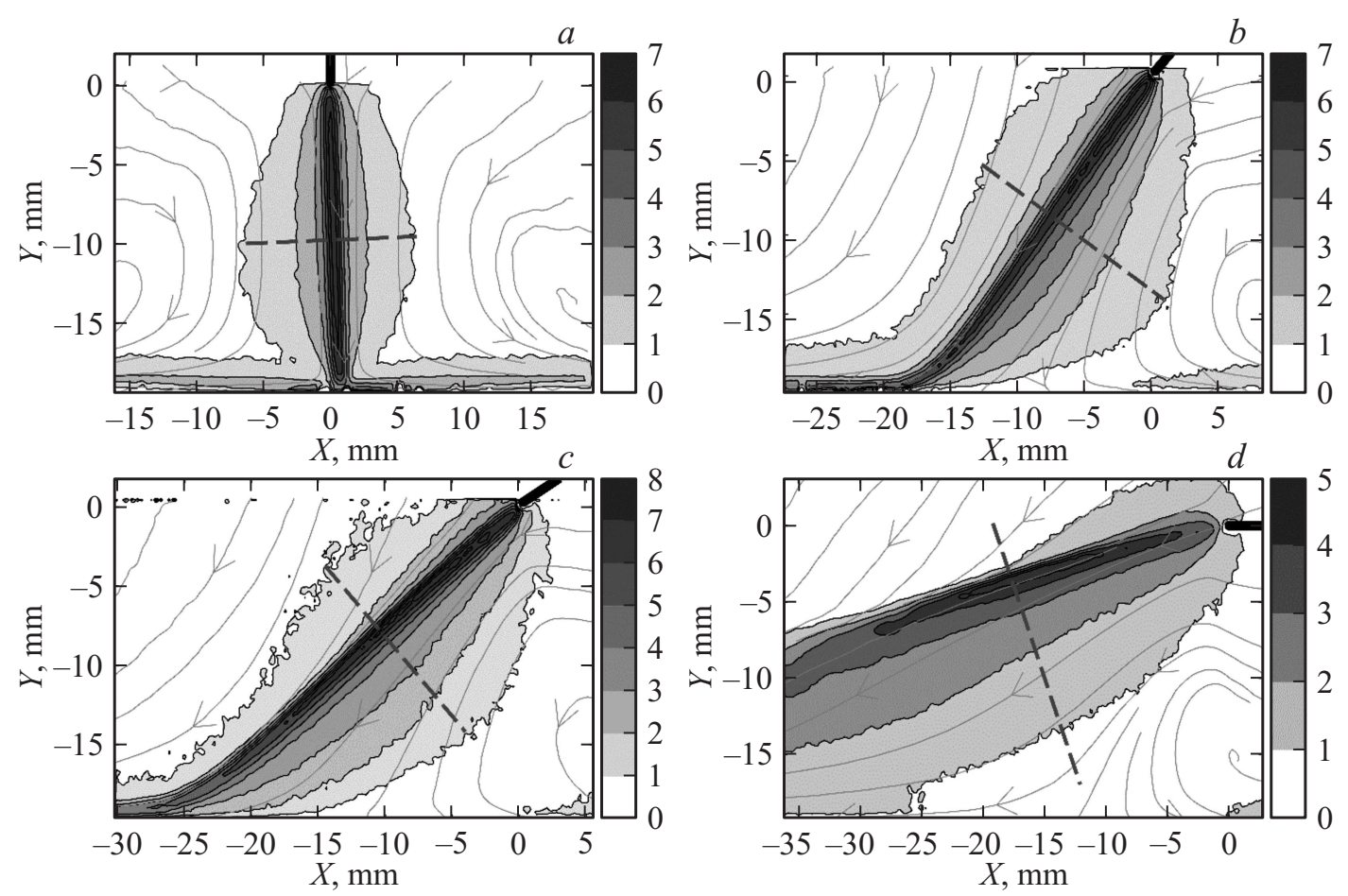

Рис. 2. Распределения скорости течений при различных углах наклона игольчатого электрода $\alpha: a-0^{\circ}, b-45^{\circ}$, $c-60^{\circ}, d-90^{\circ}$ 


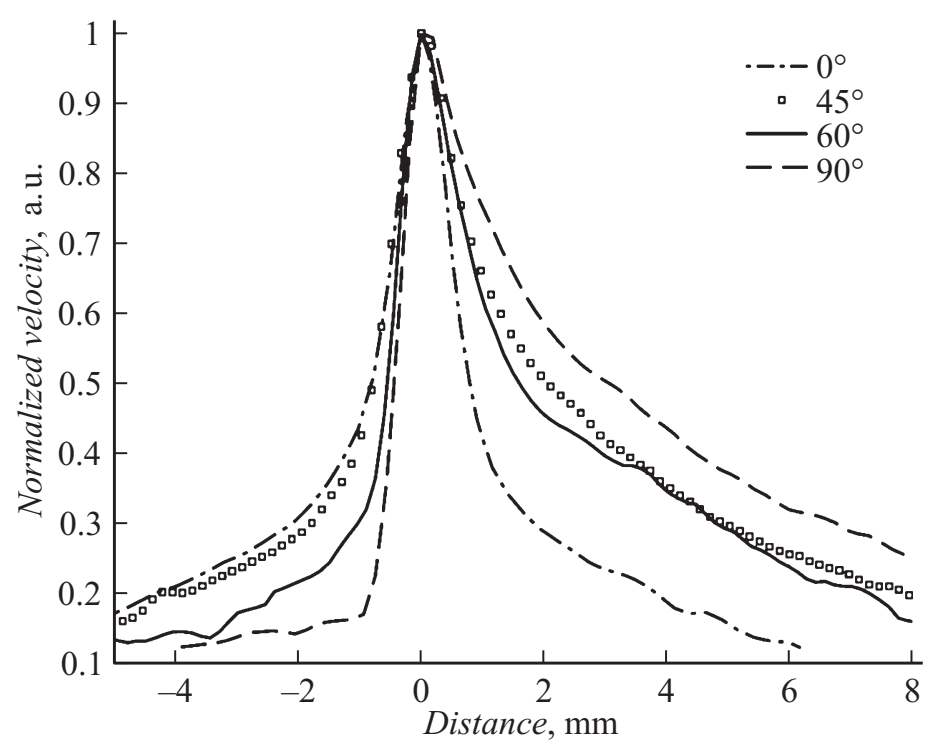

Рис. 3. Профили скорости при различных углах наклона игольчатого электрода.

углу наклона коронирующего электрода (рис. 2, $b$ и $c$ ). При горизонтальном расположении иглы (рис. $2, d$ ) струя электрического ветра немного притягивается к плоскому электроду.

Общая структура электрического ветра при наклоне иглы сохраняется, поперечный профиль течения (соответственно пунктирным линиям на рис. 2) становится шире в направлении плоского электрода (рис. 3). Размеры зоны ускорения у кончика иглы остаются малыми. Некоторые отличия можно заметить на рис. 2, $d$, где зоны ускорения и торможения стали более растянутыми в пространстве, а размер центральной области струи с максимальной скоростью уменьшился. Это связано с небольшим отклонением струи ветра относительно плоскости, на которой проводились измерения, при горизонтальном расположении иглы, когда более сильно начинают влиять процессы образования и переноса заряда на заострении иглы вблизи ее кончика. Также необходимо отметить стабильность течений в большинстве экспериментов, что характерно для положительной полярности коронирующего электрода. При проведении экспериментов с отрицательной полярностью также

Письма в ЖТФ, 2017, том 43, вып. 2 
наблюдалось изменение направления электрического ветра при наклоне иглы, однако струя была нестабильна в связи с особенностями горения отрицательной короны, что затрудняло съемку распределений скорости.

Полученные в работе данные свидетельствуют о том, что формирование струи электрического ветра происходит в основном в небольшой области около кончика иглы, электрическое поле в которой максимально. Изменение направления линий электрического поля в этой области при наклоне иглы приводит к соответствующему изменению направления действующей кулоновской силы, разгоняющей узкую струю электрического ветра. Остальная часть межэлектродного промежутка, где структура электрического поля меняется слабо, оказывает не такое сильное влияние, которое проявляется в некотором расширении профиля скорости в направлении плоского электрода. Следует отметить, что на напряженность электрического поля и на все остальные процессы также будет влиять распределение электрического заряда в межэлектродном промежутке. Этот вопрос нуждается в дополнительном исследовании, которое можно провести, например, с помощью компьютерного моделирования задачи, соответствующей эксперименту, в полной постановке с учетом распространения заряда в межэлектродном промежутке (как это делается, например, в [2]). Это, в свою очередь, является нетривиальной задачей, так как на данный момент отсутствует общепринятый подход для расчета электрического ветра с учетом всех тонкостей взаимосвязанных электрических и гидродинамических процессов. Также заметим, что учет наклона иглы приводит к необходимости использования трехмерных расчетов, что значительно увеличивает трудоемкость задачи.

В заключение необходимо отметить, что описанный выше эффект можно использовать для управления струей электрического ветра, например, при охлаждении миниатюрных объектов, которое в последнее время достаточно интенсивно исследуется [10-11] в связи с развитием и увеличением производительности микроэлектронных устройств. Также это может быть важным при создании летательных аппаратов на основе электрического ветра $[12,13]$ с целью повышения их эффективности, улучшения стабильности и управляемости. В работе [14] проанализирована роль электрического ветра в системе очистки воды от органических загрязнений. Показано, что электрический ветер существенно увеличивает скорость проникновения озона, производимого в чехле коронного разряда, в воду и, как следствие, влияет на скорость очистки

Письма в ЖТФ, 2017, том 43, вып. 2 
воды от загрязнений. При этом с помощью изменения направления электрического ветра относительно водной поверхности можно создать в воде течение оптимальной формы.

Исследования выполнены при финансовой поддержке РФФИ в рамках научного проекта № 15-08-07628 а, с использованием оборудования ресурсного центра „Геомодель“ научного парка СПбГУ.

\section{Список литературы}

[1] Верещагин И.П. Коронный разряд в аппаратах электронно-ионной технологии. М.: Энергоатомиздат, 1985. $160 \mathrm{c.}$

[2] Samusenko A., Stishkov Y., Zhidkova P. // Int. J. Plasma Environ. Technol. 2015. V. 9. N 1. P. 24-28.

[3] Moreau E. // J. Phys. D: Appl. Phys. 2007. V. 40. N 3. P. 605-636.

[4] Tsubone H., Ueno J., Komeili B. et al. // J. Electrostat. 2008. V. 66. N 1-2. P. 115-121.

[5] Ashikhmin I., Stishkov Y.K., Yakovlev V. // Int. J. Plasma Environ. Sci. Technol. 2015. V. 9. N 1. P. 13-17.

[6] Елагин И.А., Яковлев В.В., Ашихмин И.А., Стишков Ю.К. // ЖТФ. 2016. T. 86. B. 8. C. $95-101$.

[7] Niewulis A., Podliński J., Berendt A., Mizeraczyk J. // Int. J. Plasma Environ. Sci. Technol. 2014. V. 8. N 1. P. 60-71.

[8] Léger L., Moreau E., Artana G., Touchard G. // J. Electrostatics. 2001. V. 51-52. N 1-4. P. 300-306.

[9] Moreau E., Léger L., Touchard G. // J. Electrostatics. 2006. V. 64. N 3-4. P. 215-225.

[10] Wang H.-C., Jewell-Larsen N.E., Mamishev A.V. // Appl. Thermal Eng. 2013. V. 20. N 1-2. P. 190-211.

[11] Jewell-Larsen N.E., Ran H., Zhang Y. et al. // Annual IEEE Semiconductor Thermal Measurement and Management Symposium. 2009. P. 261-266.

[12] Siswanto W.A., Ngui K. // Aust. J. Basic Appl. Sci. 2011. V. 5. N 9. P. 1433-1438.

[13] Ianconescu R., Sohar D., Mudrik M. // J. Electrostatics. 2011. V. 69. N 6. P. 512-521.

[14] Takeuchi N., Takubo K. // Proceedings of the International Symposium on Electrohydrodynamics. 2014. V. 9. N 1. P. 2-6. 